\title{
Aplicación de un Modelo para el Control del Cáncer de Cuello Uterino en Soacha, Colombia
}

\author{
Applying a cervical cancer control model in Soacha, Colombia \\ Carolina Wiesner-Ceballos, Ricardo Cendales-Duarte y Sandra L. Tovar-Murillo \\ Instituto Nacional de Cancerología. Bogotá, Colombia. cwiesner@cancer.gov.co \\ acardocen@yahoo.com stovar@cancer.gov.co Universidad Externado de Colombia. Bogotá, \\ Colombia. \\ Recibido 11 Abril 2008/Enviado para Modificación 19 Octubre 2008/Aceptado 10 Noviembre 2008
}

\section{RESUMEN}

Objetivo En países de bajos ingresos, como Colombia, se ha registrado un bajo impacto en la mortalidad por cáncer de cuello uterino. En parte, este hecho se explica, por los modelos y enfoques aplicados para su control. En este estudio se aplicó el modelo para el control del cáncer propuesto por el Instituto Nacional de Cancerología que propone el desarrollo de acciones en tres niveles y el uso de estrategias sociales desde un enfoque de derechos.

Metodología Se realizó una intervención comunitaria de participación acción en tres fases: análisis de situación (2004-2005), intervención (2006) y evaluación (2007). La evaluación se realizó mediante tres componentes: análisis de las percepciones de los líderes comunitarios sobre derechos humanos. Análisis comparativo de las encuestas poblacionales; antes (2005) y después de la intervención (2007). Análisis de la demanda de servicios de citología (2001-2007). Análisis del acceso y la oportunidad al diagnostico definitivo y al tratamiento (2007).

Resultados Se encontró un incrementó significativo en las actividades relacionadas con la exigencia de derechos. Se alcanzó una cobertura del $75 \%$ en el último año entre la población más vulnerable. Se mejoró la calidad de la atención a las mujeres. La intervención recuperó la credibilidad en los servicios según tipo de afiliación al cual las mujeres tienen derecho.

Conclusión El modelo para el control del cáncer promueve el desarrollo de acciones multinivel que son efectivas. Es pertinente promover el enfoque de derechos humanos para el abordaje del control del cáncer.

Palabras Clave: Cáncer de cuello uterino, control, participación comunitaria, derechos humanos (fuente: DeCS, BIREME).

\section{ABSTRACT}

Objective Low-income countries, like Colombia, have recorded a low impact on mortality rates due to cervical cancer. This can be partially explained by the cancer control models and their approaches implemented so far. This study adopted a 
cancer control model designed by the National Cancer Institute (Instituto Nacional de Cancerología) based on interventions at three different levels and on using social strategies founded on a human rights' perspective.

Methods A participatory community-based intervention was carried out in three phases: diagnosis (2004-2005), intervention (2006) and evaluation (2007). The evaluation phase included three components: analysing community leaders' perceptions of human rights, a comparative analysis of population surveys before (2005) and after the intervention (2007) and analysing the demand for cytology services (2001-2007) and analysing gaining access to definitive diagnosis and treatment (2007).

Results A significant increase in activities related to human rights' claims was found. Cytological coverage during the last year reached $75 \%$ in the most vulnerable females. The intervention contributed towards recovering credibility in public health services. It also enhanced the quality of the services being offered to women.

Conclusion The cancer control model promoted the development of effective action at multiple levels. Encouraging a human rights' approach is pertinent in cancer control programmes.

Key Words: Cervical neoplasm, control, consumer participation, human rights, mass screening (source: MeSH, NLM).

$\mathrm{E}$ n el mundo, el carcinoma de cuello uterino es el segundo tipo de cáncer que afecta con mayor frecuencia a las mujeres, especialmente en los países con bajo desarrollo económico (1). En la mayoría de estos países, los programas para la prevención y el diagnóstico precoz de cáncer de cuello uterino no han logrado reducir la mortalidad por esta causa (2). Dentro de las razones que han explicado este hecho se encuentran: los límites de los modelos aplicados (3), el insuficiente alcance de los programas organizados de tamización (4) y el bajo rendimiento de la citología de cuello uterino en términos de su sensibilidad (5).

En el mundo, se ha manifestado la necesidad de implementar modelos de control de cáncer que tengan una perspectiva más amplia que la biomédica o la higienista, centradas en acciones individuales o en los servicios de salud (6); igualmente se ha propuesto el desarrollo de modelos que incluyan un enfoque desde las ciencias sociales como complemento a la perspectiva epidemiológica (3). A este respecto, el Instituto Nacional de Cancerología (INC), diseñó un modelo para el control de cáncer, que propone realizar acciones en el ámbito político, comunitario y en los servicios de salud mediante la utilización de estrategias sociales $(8,9)$; particularmente propone que la comunicación y educación tengan un enfoque promocional de la salud y de derechos humanos para fortalecer, en las personas marginadas y excluidas de la sociedad, las demandas de acceso a los recursos sociales, políticos y económicos. 
Los programas organizados de tamización deben cumplir varias condiciones: primero (4): alcanzar una cobertura de tamización del 80\% en la población objeto; segundo, garantizar el seguimiento de las mujeres hasta el diagnostico definitivo y tratamiento; tercero, debe tener un sistema de control de calidad, y debe hacer un monitoreo de la población intervenida en términos de incidencia y mortalidad. En Colombia, en al año 2005, se encontró que el 76,5 \% de las mujeres entre 25 a 69 años se había realizado la citología en los últimos tres años y, que las mujeres con ausencia de toma reciente no tenían seguro de salud o tenían una afiliación con subsidio (10). Igualmente se ha encontrado que dentro del sistema general de seguridad social no se hace seguimiento a las mujeres con anormalidad citológica $(11,18)$ y que el país no cuenta con un sistema para el control de calidad externo de las citologías, ni para el registro del estadio del diagnóstico de cáncer.

De acuerdo con lo anteriormente expuesto, se consideró necesario realizar un estudio en un municipio colombiano con alta concentración de población sin afiliación o afiliadas al régimen subsidiado en donde fuera posible aplicar el modelo para el control del cáncer (7), que incluyera estrategias sociales con perspectiva de derechos. Los objetivos específicos del control del cáncer se definen, de acuerdo con el modelo, a partir de un análisis de la situación a intervenir.

\section{MATERIALES Y MÉTODOS}

Se realizó una intervención comunitaria, con enfoque de participación acción y metodología cualitativa y cuantitativa en tres fases: análisis de situación, implementación y evaluación. La participación se logró con las autoridades locales municipales y departamentales de salud, con las organizaciones comunitarias y con líderes del sector salud.

Fase de análisis de situación

\section{a. Análisis cualitativo}

Se estudiaron las representaciones sociales de la citología, en las mujeres de Soacha, así como las barreras y motivaciones para su realización; igualmente se analizó la organización de los servicios de salud. La metodología y los resultados se reportan en dos artículos independientes $(11,12)$.

\section{b. Análisis cuantitativo}

b.1. Encuesta poblacional. Se estableció una línea de base mediante una encuesta poblacional a mujeres entre 25 y 69 años de edad, residentes en dos 
comunas de Soacha que difieren en sus características socio-demográficas. El universo estaba constituido por el total de manzanas existentes en cada comuna. Se hizo un muestreo probabilístico, aleatorio simple, por conglomerados y fueron encuestadas todas las mujeres residentes en las manzanas seleccionadas. Se calculó el factor de expansión de manera que las 4540 mujeres definidas en la muestra estarían representando 12,930 mujeres en el universo. Los coeficientes estimados de variación -CVE- se calcularon de acuerdo con lo planteado en el libro de texto de Särndal (13). La muestra se estimó para lograr CVE inferiores al $20 \%$ en dominios de tamaño del $15 \%$ y para estimaciones del $15 \%$ como mínimo.

b.2. Caracterización del uso de los servicios de salud. Se recogió información auditada sobre la demanda mensual de citologías según tipo de institución (registros desde el 2001 al 2007) para analizar mediante serie de tiempo. Las citologías realizadas se totalizaron diferenciando las privadas de las públicas del nivel departamental (Hospital -MGY-) y del nivel municipal (ESE). Los datos se digitaron en una base de datos validada en Epi-info 6.04d los cuales se exportaron a un archivo plano y se analizaron de manera conjunta con el software SPSS 14.0 y Decision Time 1.1.

Intervención. Del análisis de situación se encontraron tres problemas: 1) No hay un programa organizado de tamización (11); 2) Aunque la cobertura de citologías era adecuada (encuesta 2005 sin publicar), se identificaron problemas de calidad, acceso y oportunidad en los servicios (12) la encuesta 2005 mostró que las mujeres preferían usar los servicios privados antes que la red de servicios públicos a los que tienen derecho. Identificados estos problemas se propuso la intervención aplicando el modelo para el control del cáncer (7) que plantea acciones en tres ámbitos:

a. Ámbito político: Para organizar el programa de tamización se realizaron mesas de trabajo con las autoridades locales, se definieron normas técnicas y administrativas (14), se ajustaron procesos, procedimientos y rutas críticas de información; para mejorar la oportunidad en la entrega del reporte de citologías se apoyó la iniciativa local de crear un laboratorio de lectura de citologías; para mejorar de oferta de servicios para diagnóstico definitivo y se donó un colposcopio.

b. Ámbito comunitario: Para promover los procesos de construcción de ciudadanía y desarrollo local se diseñó un curso teórico práctico de 36 horas, con el apoyo del Centro de Estudios Escuela para el Desarrollo (CESDE) y Corpora- 
ción para la educación el desarrollo y la investigación popular (CEDINS). Se capacitaron 32 líderes de los diferentes barrios de cada comuna que debían replicar el proceso adquirido. Se difundió una estrategia de comunicación que incluyó un comercial de televisión, una cuña radial y actividades locales con un mensaje central basado en el derecho a recibir una atención oportuna y de calidad en los servicios de salud particularmente en caso de presentar una anormalidad citológica.

c. Ámbito de servicios de salud: Para sensibilizar a los funcionarios de salud sobre la importancia de la calidad y oportunidad de la atención se produjo y presentó una obra de teatro titulada "Tiempo y fotocopias". Posteriormente se les invitó a un curso teórico práctico de actualización en control del cáncer de cuello uterino y en estrategias para mejorar las habilidades de comunicación en salud el cual tuvo una duración de 20 horas.

Tercera fase: evaluación de la intervención

a. Evaluación cualitativa. Se definió como población participante los líderes de Soacha. La pregunta que orientó el estudio fue ¿Que percepción tienen los líderes comunitarios respecto a la relación de derechos humanos con el cáncer de cuello uterino? ¿Tuvo el curso de derechos humanos algún impacto en el municipio? ¿Qué nivel de recordación tuvo la estrategia de comunicación desde el enfoque de derechos?

Para responder a estas preguntas, se realizaron cuatro grupos focales (mayo a julio; 2007) con líderes comunitarios (13 hombres y 39 mujeres) distribuidos así: dos con población intervenida y dos con líderes de diferentes comunas. Los grupos focales fueron desarrollados por una profesional que no pertenecía al grupo de investigación para evitar sesgos en la recolección de la información. Se llevó un registro sonoro en medio magnético el cual se transcribió para su análisis. Se solicitó consentimiento verbal para la grabación y se garantizó la confidencialidad de la información. Los textos fueron guardados en formato.txt para ser procesados mediante el software Atlas-ti; mediante el cual se realizó análisis de contenido.

b. Evaluación cuantitativa

b.1 Encuesta poblacional post intervención. Se aplicó una encuesta con la misma muestra seleccionada y la misma metodología utilizada antes de la intervención. Se compararon los resultados de las dos encuestas (2006-2007). Se evaluó el incremento en la práctica de citología en la población más vulnerable, 
el uso de los servicios, la práctica del autocuidado y el acceso a la citología gratuita. Se incluyeron tres preguntas adicionales sobre exigibilidad de derechos para comparar actividades realizadas antes (2005) y durante la intervención (2006).

a.2 Caracterización del uso de los servicios de salud. Se continuó con la recolección de datos sobre demanda de citologías según tipo de Institución tres meses después de la intervención y se hizo el análisis para todo el periodo recolectado: 2001-2007. Para el análisis se utilizó el autocorrelograma y la prueba de Ljung-Box. Se eliminó a una institución privada debido a que no se pudieron auditar los registros de tomas de citologías realizadas durante el periodo 2004 - 2005. Para cada serie se ajustaron varios modelos teóricos: un modelo exponencial simple, un modelo aditivo de Winters y un modelo ARIMA con y sin intervención y se seleccionó el que más se ajustaba a los datos. Se forzaron modelos ARIMA de intervención, pero el parámetro asociado a la intervención no resultó significativo para ninguno de los tres prestadores, por lo que se decidió emplear un modelo teórico en el que no se considerase la intervención.

a.3 Evaluación del acceso y oportunidad al diagnostico definitivo y al tratamiento. Se construyó el universo de pacientes a partir de los reportes citológicos de lesión de alto grado -LEIAG- hasta carcinoma invasor registrados entre junio de 2006 y junio de 2007 en los laboratorios. Se identificaron 136 pacientes. Se decidió hacer una aproximación censal al universo de datos pues una muestra no resultaría eficiente en este caso, considerando la alta probabilidad de pérdidas. Las pacientes se contactaron tres meses después de la fecha del reporte citológico tiempo suficientes para que hubiesen tenido atención. Se emplearon medidas de tendencia central y dispersión para la descripción de las variables numéricas y frecuencias y proporciones para la descripción de las variables categóricas.

\section{RESULTADOS}

Se presentan los resultados de la intervención comunitaria y de los servicios de salud, los cuales reflejan de manera parcial la organización del programa.

El control del cáncer de cuello uterino y la perspectiva de derechos humanos

El tema de los derechos humanos ha sido trabajado en Soacha desde la última década por organizaciones no gubernamentales de manera que el curso suministrado y la estrategia de comunicación social complementaron este pro- 
ceso. Los líderes comunitarios que participaron en los grupos focales consideran que la perspectiva de derechos permite alcanzar el bienestar y mejorar las condiciones de vida así como acceso a la educación y a los servicios de salud que están relacionados con el control de cáncer de cuello uterino. Según los participantes, los derechos permiten además, la posibilidad de decidir respecto a la vida, el cuerpo y la sexualidad (Tabla 1).

Tabla 1. Textos de los líderes comunitarios de Soacha

\begin{tabular}{|c|c|}
\hline No & Textos en los grupos focales \\
\hline 1 & $\begin{array}{l}\text { Thay muchas clases de derechos, por lo menos el pr incipal es el derecho a la vida, a la salud, a } \\
\text { la educación, a una vivienda digna, a tener atención y a tener los hijcs que quiera". }\end{array}$ \\
\hline 2 & 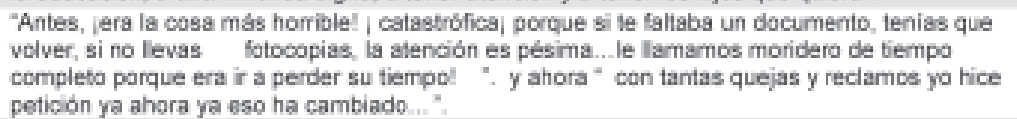 \\
\hline 3 & $\begin{array}{l}\text { "uno puede tener muy buena voluntad de servirle a la com unided pero se requieren recursos y } \\
\text { apoyo por parte de las instituciones y por parte del Estado". }\end{array}$ \\
\hline 4 & $\begin{array}{l}\text { "es un puebio y la llamada llega a una tienda y mandan a la niria para que la avisen a la } \\
\text { muchacha que esta con la enfermedad y corre la muchacha a recibir la llamada...". }\end{array}$ \\
\hline 5 & $\begin{array}{l}\text { Tay uno que pasan mucho con esta virreina Paula Andrea Betancourt, lo pasa muy seguido, } \\
\text { en el ascensor Ahi esta el esposo companero de ela que le esta motivando para que se haga la } \\
\text { chitologia y que reclame el resultado". }\end{array}$ \\
\hline
\end{tabular}

Para los participantes, los derechos humanos son una condición dada a los ciudadanos a través de la Constitución. Los derechos definen las relaciones entre los individuos y los colectivos con el Estado. Explicaron que los derechos y los deberes tienen una relación en doble vía, inseparable, en donde no se pueden comprender los unos sin los otros. Lo individual es sinónimo de "lo que a cada persona le toca hacer". El nivel colectivo significa buscar de manera conjunta frente al Estado la resolución de problemas comunes. Para ellos las problemáticas que afectan el bienestar en Soacha, se explican por el abandono del Estado así como por la falta de organización de las personas pues "No hay unión".

Los participantes asocian la salud con la presencia de los servicios públicos y percibieron un cambio en los de salud (Tabla 1) Identifican los mecanismos para garantizar el cumplimiento de los derechos al acceso de los servicios como el reclamo directo, los derechos de petición, y la tutela. La intervención motivó a algunos de los participantes a fundar una oficina de asesoría y apoyo a los usuarios de los servicios de salud. Esta oficina funcionó poco tiempo debido a problemas económicos (Tabla 1).

La mayoría de las mujeres conoce el concepto del auto-cuidado y se hace la citología. Se encontró un mejor conocimiento sobre el Virus del Papiloma Humano (VPH) en comparación con los grupos focales antes de la interven- 
ción (13). En el grupo no intervenido, los participantes confundían con mayor frecuencia el VPH con el Virus de Inmunodeficiencia Humana (VIH).

La comunidad estuvo expuesta al comercial sobre exigencia de derechos. (Tabla No 1:4) pero solo lo recordaron cuando se les mostró nuevamente durante la sesión. En contraste, el comercial que promovía la práctica de citología, producido por la Secretaria Distrital de Salud, en convenio con el Fondo de las Naciones Unidas, tuvo un alto nivel de recordación pues la comunidad admira a Paula Andrea Betancourt (Tabla No 1:5), protagonista de la estrategia.

Análisis comparativo de las encuestas poblacionales

Las características sociodemográficas de las mujeres encuestadas se muestran en la Tabla 2. La encuesta permitió hacer triangulación de algunos de los resultados encontrados en los grupos focales. Es así como se observó un incrementó significativo de actividades relacionados con la exigencia de derechos particularmente en actividades que implican recolección de firmas, reclamos en ventanillas y quejas realizadas en las oficinas de atención al usuario (Tabla 3).

Tabla 2. Características socio demográficas de las mujeres encuestadas en Soacha, según comuna de residencia. 2005

\begin{tabular}{|c|c|c|c|c|}
\hline \multirow[b]{3}{*}{ Edad } & \multicolumn{4}{|c|}{ Comuna } \\
\hline & \multicolumn{2}{|c|}{ Dos } & \multicolumn{2}{|c|}{ Cuatro } \\
\hline & No & $\%$ & No & $\%$ \\
\hline $25-35$ años & 1920 & 28,4 & 2349 & 38,0 \\
\hline $36-45$ aก๊os & 2215 & 32,8 & 2002 & 32,4 \\
\hline $46-55$ aก̃os & 1453 & 21,5 & 1101 & 17,8 \\
\hline $56-69$ años & 1164 & 17,2 & 726 & 11,8 \\
\hline Total & 6752 & 100,0 & 6178 & 100,0 \\
\hline \multicolumn{5}{|c|}{ Nivel educativo } \\
\hline Primaria & 1980 & 29,3 & 4188 & 67,8 \\
\hline Secundaria & 3566 & 52,8 & 1896 & 30,7 \\
\hline Técnico & 628 & 9,3 & 51 & 0,8 \\
\hline Universitario & 578 & 8,6 & 43 & 0,8 \\
\hline Total & 6752 & 100,0 & 6178 & 100,1 \\
\hline \multicolumn{5}{|c|}{ Afiliación al sistema de salud } \\
\hline Contribuyente & 3904 & 57,8 & 1366 & 22,1 \\
\hline Subsidiado & 2274 & 33,6 & 4112 & 66,6 \\
\hline Sin seguro & 573 & 8,4 & 700 & 11,3 \\
\hline Total & 6751 & 100,0 & 6178 & 100,0 \\
\hline
\end{tabular}


En el 2005, más del 81,0 \% de las mujeres se había realizado la citología en los últimos tres años lo que demostraba que las mujeres cumplían con su deber. Los cambios relacionados con la intervención se midieron en relación con la práctica de la citología en el último año la cual se incrementó en un 27,0 \% en la población más vulnerable (comuna cuatro).

En el año 2005, la mayoría de las mujeres se realizaban la citología en servicios privados de salud y no hacían uso de la red de servicios del seguro de salud en donde la citología es gratuita. En el año 2007, este porcentaje se invirtió y se logró que más del 95,0 \% de las mujeres de ambas comunas hicieran uso de la red de servicios del seguro de salud (Tabla 4).

Tabla 3. Comparación del número y proporción de mujeres que participaron en actividades para pedir mejoras en los servicios públicos en Soacha antes y después del año 2006, según comuna

\begin{tabular}{|c|c|c|c|c|c|c|c|c|c|c|c|c|}
\hline \multirow{3}{*}{$\begin{array}{c}\text { Actividades para } \\
\text { exigencia de } \\
\text { derechos }\end{array}$} & \multicolumn{6}{|c|}{ Comuna 2} & \multicolumn{6}{|c|}{ Comuns 4} \\
\hline & \multicolumn{3}{|c|}{ Antes del 2005} & \multicolumn{3}{|c|}{2006} & \multicolumn{3}{|c|}{ Antes det 2006} & \multicolumn{3}{|c|}{2006} \\
\hline & No & $\%$ & & No & $\%$ & & No & $\%$ & & No & & \\
\hline En marchas & 300 & 2,5 & 7.4 & 478 & 4 & 7,6 & 360 & 2,3 & 5.3 & 541 & 3,5 & 4,3 \\
\hline En cierte de vias & 77 & 0,6 & 14,3 & 146 & 1,2 & 9,2 & 265 & 1,7 & 6.5 & 282 & 1,8 & 6,1 \\
\hline $\begin{array}{l}\text { Recoleceikn de } \\
\text { fimss }\end{array}$ & 569 & 4.8 & 7.4 & 1096 & 9.2 & 3,7 & 1158 & 7.4 & 4.8 & 2138 & 13,7 & 3 \\
\hline $\begin{array}{l}\text { Reclamos por } \\
\text { vertanilla }\end{array}$ & 297 & 2,5 & 6,7 & 869 & 7,3 & 4 & 625 & 4 & 4.6 & 1454 & 9,4 & 4,1 \\
\hline $\begin{array}{l}\text { Quejas de } \\
\text { atención }\end{array}$ & 461 & 3,9 & 5,4 & 992 & 8.3 & 3,7 & 757 & 4.9 & 5.6 & 1355 & 8.7 & 3,6 \\
\hline $\begin{array}{l}\text { Reclamos buzón } \\
\text { de quejas }\end{array}$ & 196 & 1,6 & 8,7 & 389 & 3,3 & 6,9 & 236 & 1,5 & 7,6 & 464 & 3 & 9 \\
\hline $\begin{array}{l}\text { Emiso de cartes } \\
\text { a directives }\end{array}$ & 198 & 1.7 & 8.1 & 339 & 2,8 & 6.1 & 412 & 2.6 & 8.6 & 758 & 4.9 & 4.5 \\
\hline $\begin{array}{l}\text { Derechos de } \\
\text { petioión }\end{array}$ & 230 & 2 & 7 & 404 & 3,4 & 6,8 & 293 & 1,9 & 7 & 634 & 4,1 & 4,9 \\
\hline $\begin{array}{l}\text { Acciones de } \\
\text { tulata }\end{array}$ & 69 & 0,6 & 12.8 & 115 & D & 11,8 & 140 & 0.9 & 9.9 & 246 & 1.6 & 7,4 \\
\hline $\begin{array}{l}\text { Acciones de } \\
\text { cumplimiento }\end{array}$ & 44 & 0,4 & 19,6 & $\therefore$ & $=$ & - & 119 & 0.8 & 10,4 & $\therefore$ & $\therefore$ & . \\
\hline No participación & 9453 & 79,4 & $\cdot$ & 7064 & 60,4 & - & 11189 & 71,9 & $*$ & 7672 & 49,3 & - \\
\hline Total & $11 \theta 12$ & . & - & 11912 & - & - & 15554 & - & - & 15554 & - & . \\
\hline
\end{tabular}

En el año 2005 el 57,0 \% de las mujeres se habían hecho la citología por una orden médica y solo el 37,0 \% por autocuidado. En el año 2007, este último porcentaje se incremento a 45,0 \% Los resultados mostraron CVE menores al 10,0 \%. El pago o compra de insumos para la toma de citologías disminuyó de manera importante pues en el año 2005, un 19,0 \% de las mujeres pagó alguna suma y en el año 2007 solo el 6,7 \% de las mujeres lo hizo. Los resultados mostraron CVE menores al 10,0 \%.

Análisis de la demanda de servicios de toma de citología

Entre el 2001 y el 2004, había 21 instituciones que tomaban citología en Soacha: 5 privadas, 6 públicas del municipio y 10 públicas del orden departamental. Posteriormente, en los años 2004-2005 se comenzó a prestar el servicio en 2 
Tabla 4. Proporción de mujeres que se realizaron la citología en Soacha en el último año por tipo de servicio médico. Comparativo 2005-2007. Comuna 4

\begin{tabular}{clcccc}
\hline \multirow{2}{*}{ Comuna } & Tipo de servicio usado & \multicolumn{2}{c}{2005} & \multicolumn{2}{c}{2007} \\
\cline { 3 - 6 } & para la toma de citologia & No & $\%$ & No & $\%$ \\
\hline \multirow{3}{*}{ Comuna 2 } & Afiliación al SGSSS & 101 & 6,7 & 4029 & 95,4 \\
& Servicios privados & 1417 & 93,3 & 196 & 4,6 \\
& Total & 1518 & 100,0 & 4225 & 100,0 \\
& Afiliación al SGSSS & 128 & 8,3 & 4922 & 96,4 \\
Comuna 4 & Servicios privados & 1422 & 91,7 & 184 & 3,6 \\
& Total & 1550 & 100,0 & 5106 & 100,0
\end{tabular}

instituciones públicas del nivel departamental y se dejó de prestar el servicio por parte del grupo extramural; también se comenzó a prestar el servicio en siete instituciones privadas y dos IPS privadas se fusionaron. En la Figura 1 se muestra la tendencia generalizada al incremento en la demanda del número de citologías. Cabe destacar el marcado descenso que ocurrió en los meses de octubre, noviembre y diciembre de 2004 ocasionado por el cierre temporal y la posterior fusión de dos prestadores privados; dado que esta disminución fue consecuencia de la fusión descrita se decidió imputar los datos de octubre noviembre y diciembre haciendo uso de un modelo estacional simple. La disminución en el número de citologías realizadas por las entidades públicas municipales entre junio de 2002 y enero de 2003 no tiene una causa aparente por lo que no se imputan. La intervención formal del grupo investigador ocurrió a partir de junio de 2006, pero la presencia del grupo investigador desde enero del año 2005 parece haber causado un incremento en la oferta en las IPS públicas; sin embargo, no parece haber ocurrido un cambio estructural en la serie de tiempos.

El análisis individual para cada serie demuestra un incremento consistente en el número de citologías tomadas. Por medio del autocorrelograma y la prueba de Ljung-Box se demostraron autocorrelaciones significativas desde el primero hasta el décimo orden en todas las IPS lo cual confirma la tendencia al incremento ya descrita. El análisis definitivo no sugiere un efecto significativo de la intervención aunque sí se demuestra un aumento sostenido de la demanda de servicios dado el carácter progresivo de la intervención: Los resultados podrían tener un efecto sostenido durante un largo plazo que no pudo ser comprobado dado el corto período de seguimiento posterior a la intervención.

Análisis del acceso y la oportunidad al diagnostico definitivo y el tratamiento en mujeres con anormalidad citológica

Para la realización de la encuesta no se lograron ubicar 56 pacientes (41,1 \%); 2 no pertenecían al universo de estudio, 2 fallecieron y 2 rechazaron partici- 
par en

el estudio. Se hicieron entonces 74 encuestas efectivas (54,4 \%). Este bajo porcentaje de cumplimiento muestra un sesgo de selección.

Figura 1. Toma de citología en entidades públicas y privadas del municipio de

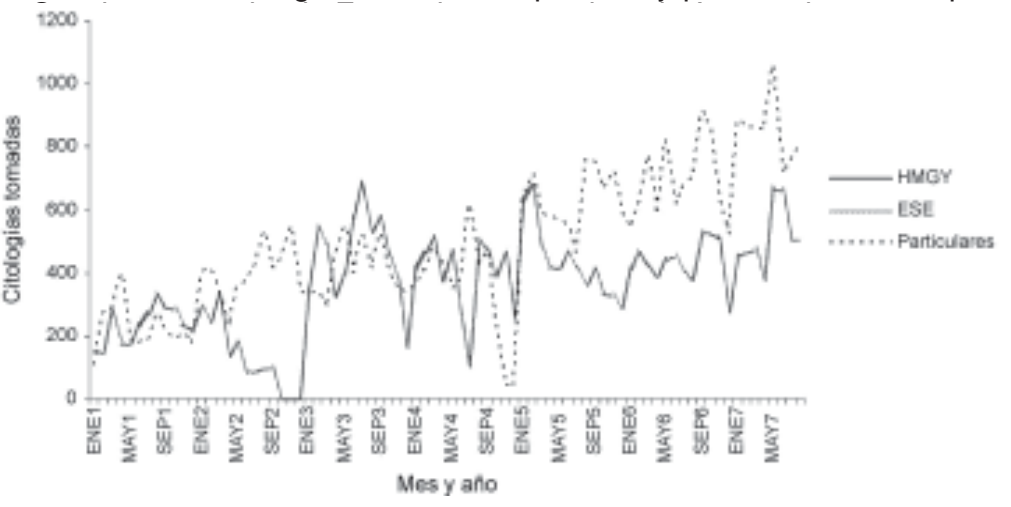

La gran mayoría de mujeres con lesiones de alto grado o cáncer invasor pudieron acceder a los servicios de colposcopia (88 \%) y biopsia (77 \%). La oportunidad en la entrega de los resultados de la biopsia superó el mes en solo en el $25 \%$ de los casos.

En el momento de la encuesta, sólo la mitad de las pacientes había recibido tratamiento por razones relacionadas con los servicios (28,0\%); aunque en un porcentaje importante de los casos estas razones estuvieron relacionadas con las mujeres (11\%). El tiempo de espera para el tratamiento fue superior a un mes en el 64 \% de los casos. La razón principal para la falta de oportunidad fueron causas administrativas relacionadas con los servicios.

\section{DISCUSIÓN}

Este estudio muestra el efecto de la aplicación del modelo para el control de cáncer, diseñado por el Instituto Nacional de Cancerología (7) y aplicado a un problema específico, como es la tamización para cáncer de cuello uterino en un municipio con un alto porcentaje de población socialmente vulnerable. De acuerdo con el modelo se debe partir de un análisis de situación, o de la información generada a partir de la investigación o de la vigilancia. El análisis de situación realizado en Soacha permitió tener claridad sobre la situación de las mujeres (12) y la organización de los servicios de salud (11). En relación con las mujeres se encontró que ellas cuentan con recursos conceptuales fren- 
te al temor que genera el cáncer de cuello uterino, gracias a que han podido hacer una adecuada objetivación de la palabra pre-cáncer y prevención (11). Esto no ha ocurrido con otras patologías malignas en donde la palabra cáncer significa sinónimo de muerte, circunstancia que se convierte en un obstáculo cultural para la promoción de la detección temprana de otras patologías como es el cáncer de mama (15).

En Soacha, incluso las mujeres más vulnerables habían cumplido con hacerse la citología en los últimos tres años a pesar de las dificultades que encontraban en la oferta de servicios de salud (11). Mediante la intervención se alcanzó una cobertura de citologías de 75\% en el último año entre la población más vulnerable. Promover la adherencia al tamizaje, debe ser un hecho que debe realizarse de manera continua en el tiempo. Estos datos son superiores a lo reportado por Profamilia en el 2005 (16) en donde se encontró una cobertura de $47,5 \%$ en el último año; es evidente que las estrategias de comunicación y educación desarrolladas desde hace ya varias décadas para la promoción de la citología han logrado penetrar la vida cotidiana de las mujeres. Sin embargo no se ha logrado disminuir el riesgo de desarrollar cáncer invasor (18)

En Soacha, las mujeres enfrentaban problemas relacionados con la calidad y oportunidad de los servicios y encontraban dificultades para tener acceso al diagnostico definitivo y al tratamiento. La ausencia de una línea de base en este aspecto, así como los sesgos de selección de las mujeres con anormalidad citológica no permiten concluir que la intervención haya mejorado la oportunidad en los tiempos de atención al diagnostico definitivo aunque se encontró una buena respuesta post intervención. Es importe que en Colombia las entidades de salud realicen mayores esfuerzos en la atención a mujeres con LEIAG, que en la cobertura de citologías (17) sobre la cual la normatividad hace énfasis $(18,19)$.

El modelo para el control del cáncer plantea que uno de los ámbitos para el desarrollo de acciones es el nivel político. Algunas dificultades encontradas para la organización de programas han sido descritas en otros estudios (20). En el actual sistema de salud hay un predominio de la racionalidad económica y la planificación se hace con base en el cumplimiento de normas (21) que hace muy rígidas las posibilidades de cambio. Es un reto para el nuevo Plan Nacional de Salud Pública (22) lograr la articulación entre los servicios de prevención con los de atención; esto implica disminuir las inequidades en el acceso a los servicios de salud de las mujeres más vulnerables del país. La aplicación del modelo del control de cáncer como el utilizado en este estudio 
puede contribuir a este objetivo. En Estados Unidos se aplicó un modelo de control de cáncer de cuello uterino que también incluye varios niveles de intervención como es el político, proveedores de servicios de salud y población que produjo un cambio significativo para mejorar la tamización en mujeres de zonas marginales urbanas (23).

Quizás el hallazgo más sobresaliente de este estudio es el cambio logrado en el uso de los servicios de salud. La intervención logró recuperar la credibilidad y el reconocimiento del valor de los servicios públicos por parte de las mujeres quienes percibían una mejor calidad en la atención en los servicios privados. Para Donabedian (25), uno de los puntos centrales de la calidad de los servicios de salud se encuentra en el grado de satisfacción por parte de los usuarios, así como la de los mismos profesionales de la salud. En Soacha, ni los usuarios ni muchos de los profesionales involucrados se sentían satisfechos con la situación respecto a la atención para el control del cáncer (11). Sin embargo, las mujeres no dejaron de utilizar los servicios de salud por encontrar una inadecuada calidad de los servicios sino que buscaron los privados que consideraron mejores independientemente que tuvieran que pagar por ello. Después de la intervención volvieron a creer en los públicos.

Esta situación es congruente con lo encontrado en una revisión sistemática (26) sobre los factores que determinan la utilización de servicios de salud. La evidencia muestra que la relación satisfacción - consumo, que plantea que a menor satisfacción, menor consumo, es en realidad inversa. Es decir, que si las personas no consiguen satisfacer sus necesidades en salud, consultan otro prestador de servicios hasta satisfacerlas. Otros estudios (25) han hecho explícito que las quejas y sentimientos de desconfianza hacia los servicios no solo es por la insuficiente capacidad técnica, sino también por la calidad del trato humano. En Latinoamérica se ha manifestado la preocupación por el débil papel que han tenido los Estados en términos de garantizar el nivel de calidad de los servicios de salud en el marco de las reforma de los sistemas de salud (28). Los resultados de este estudio muestran la necesidad de mejorar la asistencia técnica a los servicios de salud para garantizar su eficiencia y credibilidad como un elemento importante de defensa de las instituciones públicas.

Otro elemento que se destaca en este estudio es la pertinencia de promover un enfoque de derechos humanos para el control del cáncer, enfoque que en general se desconoce en el ámbito biomédico (29).Dado que la mortalidad por cáncer de cuello uterino es un indicador de inequidad en el desarrollo económico y social se hizo énfasis en la promoción de los derechos económicos, 
sociales y culturales como es el derechos a alcanzar los mayores estándares en salud, seguridad social, y el derecho a beneficiarse del progreso científico y sus aplicaciones. En conclusión se puede afirmar que el modelo para el control del cáncer promueve el desarrollo de acciones multinivel que son efectivas y que resulta pertinente promover el enfoque de derechos humanos para el abordaje del control del cáncer. Para finalizar, es importante resaltar que el enfoque de derechos humanos también hace énfasis en el hecho que las personas y las comunidades no deben ser solamente objeto de investigación sino sujetos activos para la solución de los problemas relacionados con el bienestar tanto individual como del colectivo •

Agradecimientos. Este proyecto fue financiado gracias a recursos del Instituto Nacional de Cancerología, de Colciencias, de la Universidad Externado de Colombia y la Secretaria Municipal de Salud de Soacha. Agradecemos a Yuly Salgado, enfermera en año social obligatorio, a Claudia Cortés por la realización de los grupos focales post-intervención. Al Profesor Leonardo Bautista, de la Universidad Nacional de Colombia, por la aplicación de las encuestas poblacionales. Agradecemos a las Instituciones de salud de Soacha, a la Secretaria de Salud de Cundinamarca, al Centro de Estudios Escuela para el Desarrollo CESDE y Corporación para la educación el desarrollo y la investigación popular CEDINS y a los miembros y de las organizaciones comunitarias de Soacha.

\section{REFERENCIAS}

1. Ferlay F, Bray PP, Parkin DM. GLOBOCAN 2002. Cancer Incidence. Mortality and Prevalence Worldwide IARC. Cancer Base No. 5 version 2.0 IARC Press. Lyon; 2004.

2. Sankaranarayanan R, Budukh MA, Raijkumar R. Effective screening programs for cervial cancer in low - and middle-income developing countries. Bull WHO 2001; 79(10):954961.

3. Lazcano E, Alonso O, López L, Hernandez M. Cáncer de cuello uterino: Una perspectiva histórica. Ginecol. Obstet Mex 1994; 62(2): 40-47.

4. Madlensky A, Goel V, Polzar J, Ashbury FD. Assesing the evidence for organized screening programmes. Europena J Ca 2003; 39: 1648-1653.

5. Sankaranarayanan R, Gaffikin L, Jacob M, Sellors J, Robles S. A critical assessment of screening methods for cervical neoplasia. Int J Gynaecol Obstet 2005; 89(2): 4 -12.

6. Murillo R. La prevención del cáncer más allá de las pruebas de tamizaje. Rev Col Cancerología 2003; 7(2): 23-33.

7. Murillo R, Quintero A, Piñeros M, Bravo M, Cendales R, Wiesner C, Lizcano LS. Modelo para el control del cáncer en Colombia. Bogotá: Ministerio de la Protección Social. Instituto Nacional de Cancerología. 2006.

8. Wiesner C, Cortés C, Nieto JM. Tovar S. Modelo de comunicación educativa para el control del cáncer en Colombia. Bogotá: INC; 2007.

9. Rivera-Rodriguez D, Cristancho-Cuesta A, Gonzáles-Jimenez JC: Movilización social para el control del cáncer. Bogotá. Instituto Nacional de Cancerología,2007.

10. Piñeros M, Cendales R, Murillo R, Wiesner C, Tovar S. Cobertura de la citología de cuello uterino y factores relacionados en Colombia, 2005. Rev Salud Pública 2007; 9(3):327-341. 
11. Wiesner-Ceballos C, Tovar-Murillo S, Cendales-Duarte R, Vejarano-Velandia M. Organización de los servicios de salud para el control del cáncer de cuello uterino en el Municipio de Soacha. Rev Col Cancerología 2006;10(2):98-108

12. Wiesner C, Vejarano M, Caicedo JC, Tovar S, Cendales R. La citología de cuello uterino en Soacha, Colombia: representaciones sociales, barreras y motivaciones. Revista de Salud Pública 2006; 88 (3):185-196

13. Unbiased Estimation for Cluster Sampling and Sampling in Two or More Stages. En: Särndal C, Swensson B, Wretman J. Model Assisted Survey Sampling. New York: Springer-Verlak, 1997. p. 124-154.

14. Secretaria Distrital de Soacha. Programa para el control del Cáncer de Cuello Uterino, propuesta para su organización. Soacha: Editorial Proveedora Sua; 2006.

15. Wiesner C, Cortes C, Pavajeau C, Leal MC, Tovar S. Habitus y trayectorias de riesgo: ejes articuladores en la comunicación educativa para el control del cáncer. El Caso de Patio Bonito, Bogotá. Rev Col Cancerología 2006; 10(3):155-169.

16. Ojeda G, Ordóñez M, Ochoa L, Samper B, Sánchez F. Salud sexual y reproductiva: Resultados Encuesta Nacional de Demografía y Salud 2005. Asociación Probienestar de la Familia Colombiana. Profamilia, Bienestar Familiar, United State Agency for Internacional Development, Ministerio de la Protección Social; 2005.

17. Murillo R. Programas de tamización de cáncer de cuello uterino basados en citología en América Latina. HPV Today 2007; 12:6.

18. República de Colombia, Ministerio de Salud. Resolución 412 de 28 de Febrero de 2000."Por la cual se establecen las actividades procedimientos e intervención de demanda inducida y obligatorio cumplimiento". Bogotá: Misterio de Salud; 2000.

19. República de Colombia Ministerio de Salud. Resolución 3384 de 2000. "Por la cual se modifican parcialmente las resoluciones 412 y 1745 de 2000 y se deroga la Resolución 1078 del 2000 y se establecen las metas de actividades e intervenciones de demanda inducida y obligatorio cumplimiento" Bogotá: Misterio de Salud; 2000.

20. Arbelaez M, Gaviria MB, Franco Á, Restrepo R, Hincapie D, Blas E. Tuberculosis control and managed competition in Colombia, Int J Health Plann Mgmt, 2004; (19): S25-S43.

21. Wiesner C, Murillo M, Piñeros M, Cendales R, Tovar S, Gutierrez MC. El control del cáncer en Colombia: percepciones de los funcionarios. Rev Panamericana de Salud Pública 2008. En prensa.

22. República de Colombia. Ministerio de la Protección Social. Decreto No 3039 de Agosto de 2007. "Por el cual se adopta el plan nacional de Salud Pública 2007-2010". Bogota, Ministerio de la Protección Social; 2007

23. Bastani R, Berman B, Berlin T, Crane L, Narcus A. Increasing Cervical Cancer Screening Asmong Undeserved Women in a Large Urban County Health System. Medical Car 2002; 40(10): 891-907

24. Bronfman M, Castro R, Zúñiga C, Miranda C, Oviedo J. Del "cuánto" al "por qué": la utilización de los servicios de salud desde la perspectiva de los usuarios. Salud pública Méx 1997; 39 (5)

25. Donabedian A, Attwood JC. An Evaluation of Administrative Controls in Medical Care Programs. New England Journal of Medicine, 1963; (269):347-354.

26. Mendoza R, Umberto J. Utilización de los servicios de salud: una revisión sistemática sobre los factores relacionados Cad. Saúde Pública, 2001; 17(4): 819-832.

27. Carvajal JC. La reforma de los sistemas de salud en América Latina. En: Derechos Humanos y Salud: Encontrando lazos. Memorias del Primer Taller Latinoamericano. Lima: Asociación Civil para la Educación en Desarrollo y Derechos Humanos con aplicación en Salud; 2002.

28. Ross AG, Zeballos JL, Infante A. Quality and Health Sector Reform in Latin America and the Caribbean. Revista Panamericana de Salud Pública, 2000; 8 (1-2) :93-8

29.Gruskin S, Tarantola D. Health and human rights. IN: Mc Ewen J, Detels R (Editors) Ocxford Textbook of Public Healht. Oxford: Oxford University Press, 2002;313. 